\title{
On the CFO/Channel estimation technique for MIMO-OFDM systems without using a prior knowledge of channel length
}

\author{
Tsui-Tsai Lin ${ }^{1}$ and Fuh-Hsin Hwang ${ }^{2 *}$
}

\begin{abstract}
In this paper, we propose an estimator which jointly estimates the carrier frequency offset (CFO) and channel state without a prior knowledge of channel length (CL) for a multiple-input multiple-output orthogonal frequency-division multiplexing system. A preliminary CFO/channel estimate is first reached based on the maximum-likelihood criterion. To improve the over-determined problem, a CL estimation technique is further developed. The first-order Taylor series expansion approach is used to determine the trimmed CFO estimate such that the exhaustive point search can be avoided. Simulation results have been presented to demonstrate that the proposed scheme provides a performance comparable to that of the optimal estimation which assumes that $\mathrm{CL}$ is known in advance.
\end{abstract}

Keywords: Multiple-input multiple-output (MIMO); Orthogonal frequency division multiplexing (OFDM); Carrier frequency offset (CFO); Maximum likelihood (ML); Channel length

\section{Introduction}

The multiple-input multiple-output (MIMO) scheme $[1,2]$ incorporating with the orthogonal frequency division multiplexing (OFDM) [3,4] has attracted much attention of research workers for a long while. MIMO-OFDM [5-7] becomes a key technique in wireless communications because it provides many advantages including highbandwidth efficiency, superior link quality, simple channel equalization, and resistance to the frequency-selective multipath distortion. In spite of providing these benefits, MIMO-OFDM is liable to performance degradation that is caused by some undesirable effects such as carrier frequency offset (CFO) [8-11]. This is because CFO destroys the orthogonality between subcarriers and thus reduces the detectability of OFDM symbols. In addition, the bit error rate (BER) performance of a MIMO-OFDM system is also in relation to the channel estimation accuracy $[9,10]$. Consequently, it is an important issue how we can improve the estimation accuracy of the channel for a reliable transmission, especially while CFO exists.

\footnotetext{
*Correspondence: fuhsin@nknucc.nknu.edu.tw

2 Department of Optoelectronics and Communication Engineering, National

Kaohsiung Normal University, Kaohsiung 82444, Taiwan

Full list of author information is available at the end of the article
}

To this end, in this paper, we develop an interesting technique which can jointly estimate both CFO and channel state prior to data decoding for a wireless communication system. In the past, numerous algorithms have been proposed to deal with CFO and/or channel estimation for MIMO-OFDM systems. For instance, a number of studies have been presented based on the optimal pilot design and the optimization criteria of interest for channel estimation in MIMO-OFDM systems [12-14]. In [12], a power-efficient training preamble is employed to improve the estimate complexity while the effect of CFO is considered. The Cramer-Roa lower bound (CRLB) is known to provide a lower bound on the statistical variance of any unbias estimator. In [14], the optimal pilot design and placement are determined by minimizing the CRLB in channel estimation. Zeng [15] has proposed a semiblind method for simultaneously estimating the CFO and channel of an uplink multiuser MIMO-OFDM system where a great many data are needed to estimate the subspace. In [16], a technique of joint CFO and channel estimation for uplink MIMO orthogonal frequency division multiple access (MIMO-OFDMA) systems is investigated while time-varying channels are considered. By means of the training sequence, the maximum likelihood (ML) estimator, which asymptotically achieves the CRLB, is proposed

\section{Springer}

(c) 2015 Lin and Hwang: licensee Springer. This is an Open Access article distributed under the terms of the Creative Commons Attribution License (http://creativecommons.org/licenses/by/4.0), which permits unrestricted use, distribution, and reproduction in any medium, provided the original work is properly credited. 
in [17]. Another novel ML-based scheme that estimates the aggregate effects of the channel and CFO by using two successive OFDM preambles can be found in [18]. Although the above-mentioned studies [16-18] including the ML estimate approaches $[17,18]$ provide superior performance while taking their predominance into account, all of these approaches require a prior knowledge of channel length (CL), but CL is difficult to be acquired especially when CFO is considered in MIMO-OFDM systems. When $C L$ is not known in advance or under-determined, the ML estimator's performance will not asymptotically approach the CRLB any more because the degrees of freedom are insufficient. It reveals that the knowledge of $\mathrm{CL}$ plays a very important role in $\mathrm{CFO} /$ channel estimate.

For CL estimation, a number of techniques can be found in the literatures. For example, the Akaike information criterion (AIC) [19] employs numerous data samples to represent the statistical characteristic of the channel/noise for CL estimation. However, AIC encounters over-estimate when signal-to-noise ratio (SNR) is high. The frequency-domain channel length indicator (FCLI) scheme [20] requires only one snapshot channel estimate while a prior information of noise power is assumed to be known, but FCLI becomes impractical as CFO exists. The conditional model order estimation (CME) [21] is proposed for MIMO communication channels. However, CME assumes that the CIR remains unchanged during a data frame and the processing of the block of available observations is performed offline. In [22], a joint estimate approach for channel and instantaneous CL is proposed. Unfortunately, it fails to work properly in the presence of CFO. Based on information criterion, a joint channel and $\mathrm{CL}$ estimation is proposed for single-input single-output (SISO) OFDM systems in [23] where the Levinson recursion technique is employed without significant incremental cost. Another CL estimate technique found in [24] is introduced based on the minimization of a combination of cost functions for single-input multiple-output (SIMO) channels where the channel is also assumed to be unchanged during a symbol frame. To the best of the authors' knowledge so far, no work has been devoted to the development of a joint channel and CFO estimation where the information of $\mathrm{CL}$ is not available for MIMOOFDM systems. In light of these works, it is essential to develop an approach to estimate CL especially for MIMO-OFDM systems as CFO exists.

In this paper, we aim to propose a $\mathrm{CFO} /$ channel estimation technique while achieving the estimate of CL for a MIMO-OFDM system over Rayleigh fading. The design concept of the proposed scheme is originated from the estimate technique based on the ML criterion. To determine the channel impulse response, a preliminary CFO estimate is first developed, and we define a cost function according to the ML rule by introducing the cyclic prefix
(CP) length which is more than CL. By locating the minimum value of the well-defined cost function, the CFO estimate is reached. The frequency-compensated data is then fed into the least square detector so as to compute the channel estimate while the square error function is being minimized. Although the coarse CFO and channel estimation can be obtained, the over-determined CL leads to a performance degradation. To overcome this problem, a novel CL estimation technique is derived by means of truncating the counterfeit signal paths. A fine CFO estimate employing the first-order Taylor series expansion [25] with the trimmed CL is achieved such that we can avoid employing the exhaustive point search approach. Simulation results prove the efficiency of the proposed $\mathrm{CFO} /$ channel estimation. Without using prior knowledge of $\mathrm{CL}$, the proposed scheme is shown to provide a performance comparable to that of the optimal ML estimator which assumes a perfect knowledge of CL.

This paper is organized as follows. The data model and brief preview of the ML estimation algorithm are declared in Section 2. Furthermore, the performance analysis in terms of the estimation error of CL is also included in the section. Section 3 introduces the proposed $\mathrm{CFO} /$ channel estimation for a MIMO-OFDM system without knowing the $C L$ value. Section 4 presents the computational complexity of the proposed estimator and demonstrates the comparison of complexity between the proposed and ML schemes. In Section 5, simulation results are given to verify the efficiency of the proposed scheme. Finally, Section 6 concludes the paper.

The notations used in this paper are defined as follows. Vectors and matrices are typed with boldface lower and capital letters. $\mathbf{I}_{n}$ represents an $n \times n$ identity matrix. $\mathbf{O}_{m \times n}$ is an $m \times n$ zero matrix. $\mathbf{1}_{n}$ and $\mathbf{0}_{n}$ stand for all-one and allzero vectors with length $n$, respectively. $\operatorname{diag}\left\{a_{1}, \cdots, a_{n}\right\}$ is a diagonal matrix with diagonal entries taking from $a_{1}, \cdots, a_{n} \cdot(\cdot)^{*},(\cdot)^{T}$, and $(\cdot)^{H}$ are, respectively, the complex conjugate, transpose, and Hermitian transpose of (.). $\otimes, \Re\{\cdot\}, E\{\cdot\},|\cdot|$, and $\|\cdot\|$ denote the operations taking the Kronecker product, real part, ensemble average, absolute value, and the Frobenius norm, respectively.

\section{Data model and ML estimation}

\subsection{Data model}

Let us consider an $N$-subcarrier MIMO-OFDM system deployed with $N_{t}$ transmit antennas and $N_{r}$ receive antennas in a multipath fading channel with length of $L$. The transmitted data symbols are drawn from a specified constellation set such as quadrature amplitude modulation (QAM) and then are modulated by an OFDM modulator. A CP of length $N_{g}$ is inserted into the data stream to prevent against the intersymbol interference (ISI) where $N_{g} \geq L$. At the receiving end, the received symbols are processed by the matched filtering, symbol-rate sampling, 
and CP removing in sequential order. Let a specific $N \times 1$ received data vector of the $j$ th receive antenna be given by:

$$
\begin{aligned}
\mathbf{y}_{j}= & \mathbf{E}\left(\epsilon_{0}\right) \mathbf{F}^{H} \sum_{i=1}^{N_{t}} \operatorname{diag}\left\{\mathbf{x}_{i}\right\} \mathbf{H}_{i j}+\mathbf{w}_{j} \\
= & \mathbf{E}\left(\epsilon_{0}\right) \mathbf{F}^{H}\left[\operatorname{diag}\left\{\mathbf{x}_{1}\right\}, \cdots, \operatorname{diag}\left\{\mathbf{x}_{N_{t}}\right\}\right]\left(\mathbf{I}_{N_{t}} \otimes \mathbf{F}_{L}\right) \\
& \times\left[\mathbf{h}_{1 j}^{T}, \cdots, \mathbf{h}_{N_{t} j}^{T}\right]^{T}+\mathbf{w}_{j} \\
= & \mathbf{E}\left(\epsilon_{0}\right) \mathbf{F}^{H} \mathbf{X}\left(\mathbf{I}_{N_{t}} \otimes \mathbf{F}_{L}\right) \mathbf{h}_{j}+\mathbf{w}_{j} \\
= & \mathbf{E}\left(\epsilon_{0}\right) \mathbf{A}(L) \mathbf{h}_{j}+\mathbf{w}_{j},
\end{aligned}
$$

where:

$$
\begin{aligned}
\mathbf{E}\left(\epsilon_{0}\right) & =\operatorname{diag}\left\{1, e^{j 2 \pi \epsilon_{0} / N}, \ldots, e^{j 2 \pi(N-1) \epsilon_{0} / N}\right\} \in \mathcal{C}^{N \times N} ; \\
\mathbf{h}_{j} & =\left[\mathbf{h}_{1 j}^{T}, \mathbf{h}_{2 j}^{T}, \cdots, \mathbf{h}_{N_{t} j}^{T}\right]^{T} \in \mathcal{C}^{L N_{t}} ; \\
\mathbf{X} & =\left[\operatorname{diag}\left\{\mathbf{x}_{1}\right\}, \operatorname{diag}\left\{\mathbf{x}_{2}\right\}, \cdots, \operatorname{diag}\left\{\mathbf{x}_{N_{t}}\right\}\right] \in \mathcal{C}^{N \times N N_{t}} ; \\
\mathbf{A}(L) & =\mathbf{F}^{H} \mathbf{X}\left(\mathbf{I}_{N_{t}} \otimes \mathbf{F}_{L}\right) \in \mathcal{C}^{N \times L N_{t}} .
\end{aligned}
$$

The diagonal matrix $\mathbf{E}\left(\epsilon_{0}\right)$ with $(i, i)$ th entry being $e^{j 2 \pi \epsilon_{0}(i-1) / N}$ denotes the CFO effect where $\epsilon_{0}$ is the corresponding frequency offset normalized with respect to the subcarrier spacing. In order to simplify the expressions in (1), the $N \times L N_{t}$ matrix $\mathbf{A}(L)$ is constructed by placing the $N \times L$ pre-processed data matrices $\mathbf{F}^{H} \operatorname{diag}\left\{\mathbf{x}_{i}\right\} \mathbf{F}_{L}$ for $i=1,2, \cdots, N_{t}$ side by side. $\mathbf{x}_{i}$ is the frequency-domaintransmitted symbol from the $i$ th transmit antenna. $\mathbf{H}_{i j}=$ $\mathbf{F}_{L} \mathbf{h}_{i j} \in \mathcal{C}^{N}$ is the channel frequency response where $\mathbf{h}_{i j} \in \mathcal{C}^{L}$ is the channel response between the $i$ th transmit antenna and $j$ th receive antenna. For simplicity, the path number of $N_{t r}=N_{t} N_{r}$ channels is assumed to be the same. The $N \times L$ matrix $\mathbf{F}_{L}$ is the first $L$ columns of the $N \times N$ discrete Fourier transform (DFT) matrix $\mathbf{F} \in \mathcal{C}^{N \times N}$ with the $(m, n)$ th entry being $[\mathbf{F}]_{m n}=e^{j 2 \pi m n / N} / \sqrt{N}$. Note that an $N$-point DFT is expressed as an $N \times N$ matrix multiplication as $\mathbf{X}=\mathbf{F x}$, where $\mathbf{x}$ is the input time-domain signal, and $\mathbf{X}$ is the DFT of the signal. $\mathbf{w}_{j} \in \mathcal{C}^{N}$ is the noise vector of the $j$ th receive antenna. Without loss of generality, we assume that all the entries of $\mathbf{x}_{i} \in \mathcal{C}^{N}$ are identically and independently distributed random variables with zero mean and a common variance. The received array data $\mathbf{y}=\left[\mathbf{y}_{1}^{T}, \cdots, \mathbf{y}_{N_{r}}^{T}\right]^{T} \in \mathcal{C}^{N N_{r}}$ can be expressed by:

$$
\begin{aligned}
\mathbf{y}= & {\left[\mathbf{y}_{1}^{T}, \mathbf{y}_{2}^{T}, \cdots, \mathbf{y}_{N_{r}}^{T}\right]^{T} } \\
= & {\left[\mathbf{I}_{N_{r}} \otimes \mathbf{E}\left(\epsilon_{0}\right)\right]\left[\mathbf{I}_{N_{r}} \otimes \mathbf{A}(L)\right]\left[\mathbf{h}_{1}^{T}, \cdots, \mathbf{h}_{N_{r}}^{T}\right]^{T} } \\
& +\left[\mathbf{w}_{1}^{T}, \cdots, \mathbf{w}_{N_{r}}^{T}\right]^{T} \\
= & \mathcal{E}\left(\epsilon_{0}\right) \mathcal{A}(L) \mathbf{h}+\mathbf{w},
\end{aligned}
$$

where:

$$
\begin{aligned}
\mathcal{A}(L) & =\mathbf{I}_{N_{r}} \otimes \mathbf{A}(L) \in \mathcal{C}^{N N_{r} \times L N_{t r}} ; \\
\mathcal{E}\left(\epsilon_{0}\right) & =\mathbf{I}_{N_{r}} \otimes \mathbf{E}\left(\epsilon_{0}\right) \in \mathcal{C}^{N N_{r} \times N N_{r}} ; \\
\mathbf{h} & =\left[\mathbf{h}_{1}^{T}, \mathbf{h}_{2}^{T}, \cdots, \mathbf{h}_{N_{r}}^{T}\right]^{T} \in \mathcal{C}^{L N_{t r}} ; \\
\mathbf{w} & =\left[\mathbf{w}_{1}^{T}, \mathbf{w}_{2}^{T}, \cdots, \mathbf{w}_{N_{r}}^{T}\right]^{T} \in \mathcal{C}^{N N_{r}} .
\end{aligned}
$$

\subsection{ML estimation}

For the considered system given the true CL $L$, the MLbased $\mathrm{CFO} /$ channel estimation is designed in accordance with a criterion of minimizing the log-likelihood function in terms of the unknown parameters $\mathbf{h}$ and $\epsilon_{0}$. Specifically, we can solve the following minimization problem to obtain the CFO/channel estimates [17]:

$$
\left(\hat{\mathbf{h}}, \hat{\epsilon}_{0}\right)=\arg \min _{\mathbf{h}, \epsilon}\|\mathbf{y}-\mathcal{E}(\epsilon) \mathcal{A}(L) \mathbf{h}\|^{2} .
$$

Given an $\epsilon$, optimize (5) with respect to $\mathbf{h}$, and we have the ML estimation for $\mathbf{h}$ expressed by:

$$
\hat{\mathbf{h}}=\left[\mathcal{A}^{H}(L) \mathcal{A}(L)\right]^{-1} \mathcal{A}^{H}(L) \mathcal{E}^{H}(\epsilon) \mathbf{y} \in \mathcal{C}^{L N_{t r}} .
$$

Substituting (6) into (5) and applying the linear algebraic manipulations, we have the cost function:

$$
\begin{aligned}
C(\epsilon) & =\|\mathbf{y}-\mathcal{E}(\epsilon) \mathcal{A}(L) \hat{\mathbf{h}}\|^{2} \\
& =\mathbf{y}^{H} \mathcal{E}(\epsilon) \overline{\mathbf{P}}(L) \mathcal{E}^{H}(\epsilon) \mathbf{y},
\end{aligned}
$$

where the projection matrix $\overline{\mathbf{P}}(L)$ is given by:

$$
\overline{\mathbf{P}}(L)=\mathbf{I}_{N N_{r}}-\mathcal{A}(L)\left[\mathcal{A}^{H}(L) \mathcal{A}(L)\right]^{-1} \mathcal{A}^{H}(L) \in \mathcal{C}^{N N_{r} \times N N_{r}} .
$$

It can be found that the quadratic cost function with respect to $\epsilon$ in (7) reaches a minimum value while the condition of $\epsilon=\epsilon_{0}$ is satisfied [26]. In this way, the CFO estimate can be implemented by searching the minimum of the cost function.

Because the computation of the frequency-dependent matrix $\mathcal{E}(\epsilon) \overline{\mathbf{P}}(L) \mathcal{E}^{H}(\epsilon)$, which requires $N^{2} N_{r}^{2}$ complex multiplications for each $\epsilon$, is very time-consuming, we rewrite the cost function in (7) as:

$$
C(\epsilon)=\mathbf{a}^{H}(\epsilon) \mathbf{D}_{\mathbf{y}}^{H} \overline{\mathbf{P}}(L) \mathbf{D}_{\mathbf{y}} \mathbf{a}(\epsilon),
$$

where $\mathbf{D}_{\mathbf{y}}=\operatorname{diag}\{\mathbf{y}\}$, and:

$$
\mathbf{a}(\epsilon)=\mathbf{1}_{N_{r}} \otimes\left[1, e^{j 2 \pi \epsilon / N}, \ldots, e^{j 2(N-1) \pi \epsilon / N}\right]^{T} \in \mathcal{C}^{N N_{r}} .
$$

Then, the CFO estimate is chosen by the following criterion:

$$
\begin{aligned}
\hat{\epsilon}_{0} & =\arg \min _{\epsilon} C(\epsilon) \\
& =\arg \min _{\epsilon} \mathbf{a}^{H}(\epsilon) \mathbf{D}_{\mathbf{y}}^{H} \overline{\mathbf{P}}(L) \mathbf{D}_{\mathbf{y}} \mathbf{a}(\epsilon) .
\end{aligned}
$$


This criterion only needs to compute $\mathbf{D}_{\mathbf{y}}^{H} \overline{\mathbf{P}}(L) \mathbf{D}_{\mathbf{y}} \in$ $\mathcal{C}^{N N_{r} \times N N_{r}}$ once while executing the CFO estimation, and the implementation complexity is thus greatly reduced. Note that the estimator's performance of the ML-based scheme depends on $L$. When $L$ is perfectly known, the estimator of (11) reaches the CRLB of the CFO [17].

\subsection{Performance analysis}

The ML-based estimator has been shown to achieve the CRLB when the channel length is known in advance. Inevitably, the ML approach suffers from serious performance degradation when the CL estimate is erroneous. It is interesting to evaluate the impact of CL estimation error on the MSE performance of the CFO. In this subsection, the characteristic of the estimate error in $L$ is examined. The theoretical analysis of the MSE performance associated with the ML-based estimator will be derived in terms of the CFO. Substituting $L$ with the estimate $\hat{L}$, the cost function in (7) can be rewritten by:

$$
C(\epsilon)=\mathbf{y}^{H} \mathcal{E}(\epsilon) \overline{\mathbf{P}}(\hat{L}) \mathcal{E}^{H}(\epsilon) \mathbf{y} .
$$

The ML estimator reaches the CFO estimate while the cost function is minimized. That is, the CFO estimate is derived by finding the solution for the derivative $d C(\epsilon) / d \epsilon$ being zero. Taking advantage of this feature, we analyze the MSE performance of the CFO in terms of the CL estimate.

Denote the estimated CFO as $\hat{\epsilon}_{0}=\epsilon_{0}+\Delta$ where $\Delta$ is the CFO estimation error and $\Delta \ll 1$. The first derivative of the cost function is approximated by the first-order Taylor series expansion [25] written as:

$$
\begin{aligned}
\dot{C}\left(\hat{\epsilon}_{0}\right) & =\left.\frac{d C(\epsilon)}{d \epsilon}\right|_{\epsilon=\hat{\epsilon}_{0}} \\
& \approx \dot{C}\left(\epsilon_{0}\right)+\Delta \ddot{C}\left(\epsilon_{0}\right) .
\end{aligned}
$$

Since $C(\epsilon)$ is expressed by (12), we further rewrite $\dot{C}\left(\epsilon_{0}\right)$ and $\ddot{C}\left(\epsilon_{0}\right)$ by:

$$
\begin{aligned}
\dot{C}\left(\epsilon_{0}\right)= & 2 \Re\left\{\mathbf{y}^{H} \dot{\mathcal{E}}\left(\epsilon_{0}\right) \overline{\mathbf{P}}(\hat{L}) \mathcal{E}^{H}\left(\epsilon_{0}\right) \mathbf{y}\right\} ; \\
= & 2 \Re\left\{\dot{\tilde{\mathbf{y}}}^{H} \overline{\mathbf{P}}(\hat{L}) \tilde{\mathbf{y}}\right\} ; \\
\ddot{C}\left(\epsilon_{0}\right)= & 2 \Re\left\{\mathbf{y}^{H} \ddot{\mathcal{E}}\left(\epsilon_{0}\right) \overline{\mathbf{P}}(\hat{L}) \mathcal{E}^{H}\left(\epsilon_{0}\right) \mathbf{y}\right\} \\
& +2 \mathbf{y}^{H} \dot{\mathcal{E}}\left(\epsilon_{0}\right) \overline{\mathbf{P}}(\hat{L}) \dot{\mathcal{E}}^{H}\left(\epsilon_{0}\right) \mathbf{y} \\
= & 2 \Re\left\{\ddot{\tilde{\mathbf{y}}}^{H} \overline{\mathbf{P}}(\hat{L}) \tilde{\mathbf{y}}\right\}+2 \dot{\tilde{\mathbf{y}}}^{H} \overline{\mathbf{P}}(\hat{L}) \dot{\tilde{\mathbf{y}}}
\end{aligned}
$$

where:

$$
\begin{aligned}
\tilde{\mathbf{y}} & =\mathcal{E}^{H}\left(\epsilon_{0}\right) \mathbf{y} ; \\
\dot{\tilde{\mathbf{y}}} & =\dot{\mathcal{E}}^{H}\left(\epsilon_{0}\right) \mathbf{y} ; \\
\ddot{\tilde{\mathbf{y}}} & =\ddot{\mathcal{E}}^{H}\left(\epsilon_{0}\right) \mathbf{y} .
\end{aligned}
$$

Recall that the CFO estimate $\hat{\epsilon}_{0}$ is reached while the cost function in (12) is minimized. By finding the solution of $\dot{C}\left(\hat{\epsilon}_{0}\right)=0$, the estimation error is obtained and expressed by:

$$
\begin{aligned}
\Delta & =-\frac{\dot{C}\left(\epsilon_{0}\right)}{\ddot{C}\left(\epsilon_{0}\right)} \\
& =-\frac{\Re\left\{\dot{\tilde{\mathbf{y}}}^{H} \overline{\mathbf{P}}(\hat{L}) \tilde{\mathbf{y}}\right\}}{\Re\left\{\ddot{\tilde{\mathbf{y}}}^{H} \overline{\mathbf{P}}(\hat{L}) \tilde{\mathbf{y}}\right\}+\dot{\tilde{\mathbf{y}}}^{H} \overline{\mathbf{P}}(\hat{L}) \dot{\tilde{\mathbf{y}}}} .
\end{aligned}
$$

To investigate the effect of CL estimation error, the received data are rewritten in terms of CL estimate $\hat{L}$ :

$$
\mathbf{y}=\mathcal{E}\left(\epsilon_{0}\right)[\mathcal{A}(\hat{L}) \overline{\mathbf{h}}+\mathbf{u}]+\mathbf{w},
$$

where $\mathbf{u}=u[L-\hat{L}][\mathcal{A}(L) \mathbf{h}-\mathcal{A}(\hat{L}) \overline{\mathbf{h}}] \in \mathcal{C}^{N N_{r}}$ is the residual signal term, $u[n]$ is the unit step function, and:

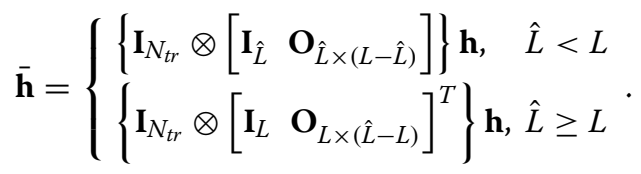

For the over-determined case $(\hat{L}>L)$, the residual signal vector $\mathbf{u}$ vanishes $(\mathbf{u}=\mathbf{0})$ and the augmented entries in $\overline{\mathbf{h}}$ are all null. Using (17) in company with the identity $\overline{\mathbf{P}}(\hat{L}) \mathcal{E}^{H}\left(\epsilon_{0}\right) \mathbf{y}=\overline{\mathbf{P}}(\hat{L}) \mathbf{v},(16)$ results as:

$$
\Delta=-\frac{\Re\left\{\left[\dot{\mathcal{E}}_{0}^{H} \mathcal{A}(\hat{L}) \overline{\mathbf{h}}+\dot{\mathbf{v}}\right]^{H} \overline{\mathbf{P}}(\hat{L}) \mathbf{v}\right\}}{\Re\left\{\left[\ddot{\mathcal{E}}_{0}^{H} \mathcal{A}(\hat{L}) \overline{\mathbf{h}}+\dot{\mathbf{v}}\right]^{H} \overline{\mathbf{P}}(\hat{L}) \mathbf{v}\right\}+\left[\dot{\mathcal{E}}_{0}^{H} \mathcal{A}(\hat{L}) \overline{\mathbf{h}}+\dot{\mathbf{v}}\right]^{H} \overline{\mathbf{P}}(\hat{L})\left[\dot{\mathcal{E}}_{0}^{H} \mathcal{A}(\hat{L}) \overline{\mathbf{h}}+\dot{\mathbf{v}}\right]},
$$

where $\mathbf{v}=\mathbf{u}+\mathcal{E}^{H}\left(\epsilon_{0}\right) \mathbf{w} \in \mathcal{C}^{N N_{r}}, \dot{\mathbf{v}}=\dot{\mathcal{E}}_{0}^{H} \mathbf{w}$, and $\ddot{\mathbf{v}}=\ddot{\mathcal{E}}_{0}^{H} \mathbf{w}$. It is clear that the estimation error in (19) depends on the value of $\hat{L}$ as well as the SNR. For sufficiently high SNR, the noise term is negligible such that the estimation error $\Delta$ can be approximated by:

$$
\begin{aligned}
& \Delta \approx-\frac{\Re\left\{\left[\mathcal{A}(\hat{L}) \overline{\mathbf{h}}+\mathbf{u}+\mathcal{E}^{H}\left(\epsilon_{0}\right) \mathbf{w}\right]^{H} \dot{\mathcal{E}}_{0} \overline{\mathbf{P}}(\hat{L})\left[\mathbf{u}+\mathcal{E}^{H}\left(\epsilon_{0}\right) \mathbf{w}\right]\right\}}{\Re\left\{[\mathcal{A}(\hat{L}) \overline{\mathbf{h}}+\mathbf{u}]^{H} \ddot{\mathcal{E}}_{0}^{H} \overline{\mathbf{P}}(\hat{L}) \mathbf{u}\right\}+[\mathcal{A}(\hat{L}) \overline{\mathbf{h}}+\mathbf{u}]^{H} \dot{\mathcal{E}}_{0} \overline{\mathbf{P}}(\hat{L}) \dot{\mathcal{E}}_{0}^{H}[\mathcal{A}(\hat{L}) \overline{\mathbf{h}}+\mathbf{u}]} \\
& =-\frac{\Re\left\{[\mathcal{A}(\hat{L}) \overline{\mathbf{h}}+\mathbf{u}]^{H} \dot{\mathcal{E}}_{0} \overline{\mathbf{P}}(\hat{L})\left[\mathbf{u}+\mathcal{E}^{H}\left(\epsilon_{0}\right) \mathbf{w}\right]+\mathbf{w}^{H} \mathcal{E}\left(\epsilon_{0}\right) \dot{\mathcal{E}}_{0} \overline{\mathbf{P}}(\hat{L})\left[\mathbf{u}+\mathcal{E}^{H}\left(\epsilon_{0}\right) \mathbf{w}\right]\right\}}{\Re\left\{[\mathcal{A}(\hat{L}) \overline{\mathbf{h}}+\mathbf{u}]^{H} \ddot{\mathcal{E}}_{0} \overline{\mathbf{P}}(\hat{L}) \mathbf{u}\right\}+[\mathcal{A}(\hat{L}) \overline{\mathbf{h}}+\mathbf{u}]^{H} \dot{\mathcal{E}}_{0} \overline{\mathbf{P}}(\hat{L}) \dot{\mathcal{E}}_{0}^{H}[\mathcal{A}(\hat{L}) \overline{\mathbf{h}}+\mathbf{u}]} \\
& \approx-\frac{\Re\left\{[\mathcal{A}(\hat{L}) \overline{\mathbf{h}}+\mathbf{u}]^{H} \dot{\mathcal{E}}_{0} \overline{\mathbf{P}}(\hat{L})\left[\mathbf{u}+\mathcal{E}^{H}\left(\epsilon_{0}\right) \mathbf{w}\right]\right\}}{\Re\left\{[\mathcal{A}(\hat{L}) \overline{\mathbf{h}}+\mathbf{u}]^{H} \ddot{\mathcal{E}}_{0} \overline{\mathbf{P}}(\hat{L}) \mathbf{u}\right\}+[\mathcal{A}(\hat{L}) \overline{\mathbf{h}}+\mathbf{u}]^{H} \dot{\mathcal{E}}_{0} \overline{\mathbf{P}}(\hat{L}) \dot{\mathcal{E}}_{0}^{H}[\mathcal{A}(\hat{L}) \overline{\mathbf{h}}+\mathbf{u}]} .
\end{aligned}
$$

Here, the performance analysis is presented by considering two distinct conditions including the over-determined and under-determined cases. 


\subsubsection{Over-determined case}

In the case of the correct or over-determined estimation, i.e., $\hat{L} \geq L$, since $\mathbf{u}=\mathbf{0}_{N N_{r}}, \Delta$ can be further simplified to the expressions as:

$$
\begin{aligned}
\Delta & \approx-\frac{\Re\left\{\overline{\mathbf{h}}^{H} \mathcal{A}^{H}(\hat{L}) \dot{\mathcal{E}}_{0} \overline{\mathbf{P}}(\hat{L}) \mathcal{E}^{H}\left(\epsilon_{0}\right) \mathbf{w}\right\}}{\overline{\mathbf{h}}^{H} \mathcal{A}^{H}(\hat{L}) \dot{\mathcal{E}}_{0} \overline{\mathbf{P}}(\hat{L}) \dot{\mathcal{E}}_{0}^{H} \mathcal{A}(\hat{L}) \overline{\mathbf{h}}} \\
& =-\frac{\Re\left\{\mathbf{h}^{H} \mathcal{A}^{H}(L) \dot{\mathcal{E}}_{0} \overline{\mathbf{P}}(\hat{L}) \mathcal{E}^{H}\left(\epsilon_{0}\right) \mathbf{w}\right\}}{\mathbf{h}^{H} \mathcal{A}^{H}(L) \dot{\mathcal{E}}_{0} \overline{\mathbf{P}}(\hat{L}) \dot{\mathcal{E}}_{0}^{H} \mathcal{A}(L) \mathbf{h}} .
\end{aligned}
$$

The second equality holds owing to $\mathcal{A}(\hat{L}) \overline{\mathbf{h}}=\mathcal{A}(L) \mathbf{h}$ because (18) guarantees that the augmented channel responses in $\overline{\mathbf{h}}$ are zero. From (20), the MSE performance of the CFO mainly depends on the channel impulse response $\mathbf{h}$ and noise. To examine the noise effect on the MSE, let $\mathbf{h}$ be given and the mean and mean square of the estimation error can be thus derived as:

$$
\begin{aligned}
E\{\Delta\} & \approx 0 ; \\
E\left\{|\Delta|^{2}\right\} & \approx \frac{E\left\{\left|\Re\left\{\mathbf{h}^{H} \mathcal{A}^{H}(L) \dot{\mathcal{E}}_{0} \overline{\mathbf{P}}(L) \mathcal{E}^{H}\left(\epsilon_{0}\right) \mathbf{w}\right\}\right|^{2}\right\}}{\left|\mathbf{h}^{H} \mathcal{A}^{H}(L) \dot{\mathcal{E}}_{0} \overline{\mathbf{P}}(\hat{L}) \dot{\mathcal{E}}_{0}^{H} \mathcal{A}(L) \mathbf{h}\right|^{2}} \\
& =\frac{\sigma_{n}^{2} \mathbf{h}^{H} \mathcal{A}^{H}(L) \dot{\mathcal{E}}_{0} \overline{\mathbf{P}}(L) \dot{\mathcal{E}}_{0}^{H} \mathcal{A}(L) \mathbf{h}}{2\left|\mathbf{h}^{H} \mathcal{A}^{H}(L) \dot{\mathcal{E}}_{0} \overline{\mathbf{P}}(\hat{L}) \dot{\mathcal{E}}_{0}^{H} \mathcal{A}(L) \mathbf{h}\right|^{2}} \\
& =\frac{\sigma_{n}^{2}}{2}\left[\mathbf{h}^{H} \mathcal{A}^{H}(L) \dot{\mathcal{E}}_{0} \overline{\mathbf{P}}(L) \dot{\mathcal{E}}_{0}^{H} \mathcal{A}(L) \mathbf{h}\right]^{-1},
\end{aligned}
$$

respectively. As expected, for $\hat{L} \geq L$, the ML-based scheme is an unbias estimator, and the MSE value of the ML estimation is increased by the noise power.

\subsubsection{Under-determined case}

If CL estimate is less than the value of $L$, i.e., $\hat{L}<L$, the residual signal $\mathbf{u}$ exists and cannot be omitted, especially for high SNR. For $\sigma_{n}^{2} \ll 1$, since $\overline{\mathbf{P}}(\hat{L}) \mathcal{A}(\hat{L})=\mathbf{O}_{N N_{r} \times N N_{r}}$, the CFO estimation error is simplified to the following expression:
The above expression indicates that the estimation error depends on both $\mathbf{h}$ and $\hat{L}$. Given $\mathbf{h}$, the $\mathrm{ML}$ estimator becomes bias $(E\{\Delta\} \neq 0)$. The ML estimator fails to achieve a reliable performance even when the SNR is increased. This implies that the ML estimation strongly depends on the accuracy of the CL estimation.

\section{Proposed joint CFO and channel estimation}

It is well known that the ML-based scheme requires the prior knowledge of $L$ to construct $\mathcal{A}(L)$. In practice, the instantaneous value of $L$ is difficult to be reached, and it should be pre-determined prior to the ML estimation. In the case of the over-determined CL, the redundant dimension of the subspace in $\mathbf{P}(L)$ leads to a large noise power, which slightly degrades the estimation performance. With regards to the under-determined CL, the ML-based estimator breaks down because of insufficiency of the degree of freedom for the channel estimation in (6). Therefore, a correct CL is essential for the ML-based estimation. In light of the aforementioned problems, it encourages us to develop a joint CFO and channel estimation, which does not require a prior knowledge of CL. Since the maximum path delay is less than the CP length, $N_{g}$, we can regard the CP length as the preliminary estimate of CL to avoid the break down of performance owing to an insufficient degree of freedom. Here, we replace $N_{g}$ with $L$ in (11), and the coarse CFO estimate is derived by:

$$
\hat{\epsilon}_{c}=\arg \min _{\epsilon} \mathbf{a}^{H}(\epsilon) \mathbf{D}_{\mathbf{y}}^{H} \overline{\mathbf{P}}\left(N_{g}\right) \mathbf{D}_{\mathbf{y}} \mathbf{a}(\epsilon) .
$$

Although the coarse CFO can be obtained, the estimator suffers slight performance degradation due to a large noise term introduced by the redundant dimension of $\mathcal{A}\left(N_{g}\right) \in$ $\mathcal{C}^{N N_{r} \times N_{g} N_{t r}}$. To alleviate the degradation, one may properly choose an $L$ from the effective channel response, which is constructed by means of truncating the estimated channel response. Based on the coarse CFO estimate in (21), the frequency-compensated data is given by $\mathcal{E}^{H}\left(\hat{\epsilon}_{c}\right) \mathbf{y}$ and the coarse channel estimate can be determined by the least square error criterion:

$$
\min _{\mathbf{h}}\left\|\mathcal{E}^{H}\left(\hat{\epsilon}_{c}\right) \mathbf{y}-\mathcal{A}\left(N_{g}\right) \mathbf{h}\right\|^{2},
$$

$$
\begin{aligned}
& \Delta \approx-\frac{\Re\left\{[\mathcal{A}(\hat{L}) \overline{\mathbf{h}}+\mathbf{u}]^{H} \dot{\mathcal{E}}_{0} \overline{\mathbf{P}}(\hat{L}) \mathbf{u}\right\}}{\Re\left\{[\mathcal{A}(\hat{L}) \overline{\mathbf{h}}+\mathbf{u}]^{H} \ddot{\mathcal{E}} 0 \overline{\mathbf{P}}(\hat{L}) \mathbf{u}\right\}+[\mathcal{A}(\hat{L}) \overline{\mathbf{h}}+\mathbf{u}]^{H} \dot{\mathcal{E}}_{0} \overline{\mathbf{P}}(\hat{L}) \dot{\mathcal{E}}_{0}^{H}[\mathcal{A}(\hat{L}) \overline{\mathbf{h}}+\mathbf{u}]} \\
&=-\frac{\Re\left\{\mathbf{h}^{H} \mathcal{A}^{H}(L) \dot{\mathcal{E}}_{0} \overline{\mathbf{P}}(\hat{L}) \mathcal{A}(L) \mathbf{h}-\mathbf{h}^{H} \mathcal{A}^{H}(L) \dot{\mathcal{E}}_{0} \overline{\mathbf{P}}(\hat{L}) \mathcal{A}(\hat{L}) \mathbf{h}\right\}}{\Re\left\{\mathbf{h}^{H} \mathcal{A}^{H}(L) \ddot{\mathcal{E}}_{0} \overline{\mathbf{P}}(\hat{L}) \mathcal{A}(L) \mathbf{h}-\mathbf{h}^{H} \mathcal{A}^{H}(L) \ddot{\mathcal{E}}_{0}^{H} \overline{\mathbf{P}}(\hat{L}) \mathcal{A}(\hat{L}) \overline{\mathbf{h}}\right\}+\mathbf{h}^{H} \mathcal{A}^{H}(L) \dot{\mathcal{E}}_{0} \overline{\mathbf{P}}(\hat{L}) \dot{\mathcal{E}}_{0}^{H} \mathcal{A}(L) \mathbf{h}} \\
&=-\frac{\Re\left\{\mathbf{h}^{H} \mathcal{A}^{H}(L) \dot{\mathcal{E}}_{0} \overline{\mathbf{P}}(\hat{L}) \mathcal{A}(L) \mathbf{h}\right\}}{\Re\left\{\mathbf{h}^{H} \mathcal{A}^{H}(L) \ddot{\mathcal{E}}_{0} \overline{\mathbf{P}}(\hat{L}) \mathcal{A}(L) \mathbf{h}\right\}+\mathbf{h}^{H} \mathcal{A}^{H}(L) \dot{\mathcal{E}}_{0} \overline{\mathbf{P}}(\hat{L}) \dot{\mathcal{E}}_{0}^{H} \mathcal{A}(L) \mathbf{h}} .
\end{aligned}
$$


whose solution is derived:

$$
\hat{\mathbf{h}}=\left[\mathcal{A}^{H}\left(N_{g}\right) \mathcal{A}\left(N_{g}\right)\right]^{-1} \mathcal{A}^{H}\left(N_{g}\right) \mathcal{E}^{H}\left(\hat{\epsilon}_{c}\right) \mathbf{y} \in \mathcal{C}^{N_{g} N_{t r}} .
$$

Note that there are a few of counterfeit signal paths since $N_{g}>L$. This implies that a truncated scheme is required where the effective paths are reserved and the other paths are eliminated. Owing to the hardware implementation limitations, the receive antennas are usually spaced closely, and the channel response for each path between a specified transmit antenna and any receive antenna is assumed to be similar. To relieve the noise effect, the effective channel response is obtained by averaging all normalized channel responses of the paths from one specified transmit antenna to all receive antennas. The normalized channel response associated with the $q$ th receive antenna is defined by:

$$
\tilde{\mathbf{h}}_{q}=\left(\mathbf{e}_{q}^{T} \otimes \mathbf{I}_{N_{g}}\right)|\hat{\mathbf{h}}| / \max \left\{\left(\mathbf{e}_{q}^{T} \otimes \mathbf{I}_{N_{r}}\right)|\hat{\mathbf{h}}|\right\} \in \mathcal{C}^{N_{g}},
$$

where $\mathbf{e}_{q}$ denotes the $q$ th column of $\mathbf{I}_{N_{t r}}$. The resulting effective channel response is expressed by:

$$
\hat{\mathbf{h}}_{\text {eff }}=\sum_{q=1}^{N_{t r}} \tilde{\mathbf{h}}_{q} / \max \left\{\sum_{q=1}^{N_{t r}} \tilde{\mathbf{h}}_{q}\right\} \in \mathcal{C}^{N_{g}} .
$$

According to (25), the estimated channel length, $\hat{L}$, can be obtained by:

$$
\hat{L}=\arg \max _{i} \hat{h}_{e f f}(i)>\gamma,
$$

where $\hat{h}_{\text {eff }}(i)$ denotes the $i$ th element of $\hat{\mathbf{h}}_{\text {eff }}$ and $\gamma$ is a preset threshold. The chosen parameter should be small enough so as to decrease the miss probability and increase the probability of detection while avoiding a high false alarm probability. More detailed discussion about the selection of $\gamma$ will be given shortly in Section 5 .

Replacing $N_{g}$ with $\hat{L}$ in (21) and using the exhaustive point search, we can obtain the trimming CFO estimate. The computational complexity of the search for CFO has relations with the number of subcarriers, paths, and transmit and receive antennas as well as the searching granularity. The smaller the granularity is, the better the estimation accuracy will be. However, decreasing the granularity will increase the computational complexity, especially when there are a great number of subcarriers. A possible solution is to utilize the first-order Taylor expansion technique associated with the preliminary CFO estimate in (21). In this way, the concave cost function can be transformed into a quadratic function with respect to the normalized CFO, in which the trimming CFO estimate achieves the minimum and can be easily obtained. Denote $\mathbf{a}_{0}=\mathbf{a}(0)$ and $\dot{\mathbf{a}}_{0}=d \mathbf{a}(\epsilon) /\left.d \epsilon\right|_{\epsilon=0}$ and we have:

$$
\begin{aligned}
\mathbf{a}\left(\hat{\epsilon}_{c}+\epsilon\right) & \approx \mathbf{a}\left(\hat{\epsilon}_{c}\right)+\epsilon \times\left.\frac{d \mathbf{a}(\epsilon)}{d \epsilon}\right|_{\epsilon=\hat{\epsilon}_{c}} \\
& =\mathcal{E}\left(\hat{\epsilon}_{c}\right)\left(\mathbf{a}_{0}+\epsilon \dot{\mathbf{a}}_{0}\right) .
\end{aligned}
$$

Substituting (27) into (9), the cost function can be rewritten by:

$$
C(\epsilon) \approx\left[\mathcal{E}\left(\hat{\epsilon}_{c}\right)\left(\mathbf{a}_{0}+\epsilon \dot{\mathbf{a}}_{0}\right)\right]^{H} \mathbf{D}_{\mathbf{y}}^{H} \overline{\mathbf{P}}(\hat{L}) \mathbf{D}_{\mathbf{y}} \times\left[\mathcal{E}\left(\hat{\epsilon}_{c}\right)\left(\mathbf{a}_{0}+\epsilon \dot{\mathbf{a}}_{0}\right)\right],
$$

which reaches the minimum as the first derivative of (28) equals to zero. Applying some linear algebraic manipulation techniques, the trimming CFO estimate is derived by:

$$
\hat{\epsilon}_{0}=\hat{\epsilon}_{c}-\frac{\Re\left\{\mathbf{a}_{0}^{H} \mathcal{E}\left(\hat{\epsilon}_{c}\right) \mathbf{D}_{\mathbf{y}}^{H} \mathbf{P}(\hat{L}) \mathbf{D}_{\mathbf{y}} \mathcal{E}^{H}\left(\hat{\epsilon}_{c}\right) \dot{\mathbf{a}}_{0}\right\}}{\dot{\mathbf{a}}_{0}^{H} \mathcal{E}\left(\hat{\epsilon}_{c}\right) \mathbf{D}_{\mathbf{y}}^{H} \mathbf{P}(\hat{L}) \mathbf{D}_{\mathbf{y}} \mathcal{E}^{H}\left(\hat{\epsilon}_{c}\right) \dot{\mathbf{a}}_{0}}
$$

Substituting $\hat{L}$ in (26) and $\hat{\epsilon}_{0}$ in (29) into (23), we have the trimming estimate of the channel response.

\section{Computational complexity}

Conveniently enough, the proposed estimator's computational complexity is evaluated by counting the approximate number of total arithmetic operations including addition and multiplication floating-point operations (flops) in the algorithm. Because the computational complexity of the proposed scheme is dominated by the processing in the coarse CFO estimation, the total complexity

\begin{tabular}{|c|c|c|c|c|c|c|}
\hline \multirow{3}{*}{ Algorithm } & \multicolumn{6}{|c|}{ Numbers of flops } \\
\hline & \multicolumn{6}{|l|}{$\left(N, N_{g}, L\right)$} \\
\hline & $(64,16,1)$ & $(64,16,5)$ & $(128,32,1)$ & $(128,32,5)$ & $(256,64,1)$ & $(256,64,5)$ \\
\hline Proposed & $1.240 \mathrm{E} 8$ & $1.240 \mathrm{E} 8$ & 4.963E8 & 4.963E8 & $1.996 \mathrm{E} 9$ & 1.996E9 \\
\hline$M L$ & $1.235 \mathrm{E} 8$ & $1.236 \mathrm{E} 8$ & 4.929E8 & 4.930E8 & 1.969E9 & 1.970 E9 \\
\hline
\end{tabular}
load can be approximated as the required number of flops for determining the solution of (21). The computation for finding the inverse of the $N_{g} N_{t r} \times N_{g} N_{t r}$ block diagonal matrix $\mathcal{A}^{H}\left(N_{g}\right) \mathcal{A}\left(N_{g}\right)$ in (21) involves [27]:

$$
\frac{1}{2} N N_{t}\left(1+N_{t}\right)\left[1+N_{g}\left(1+2 N_{g}\right)\right]+\frac{4}{3} N_{g}^{3} N_{t}^{3}
$$

Table 1 Comparison for the computational complexity between the proposed and ML schemes

$\left(N_{g}=N / 4, N_{t}=N_{r}=2, \mu=10^{4}\right)$. 
flops. In addition, constructing $\overline{\mathbf{P}}\left(N_{g}\right)$ costs about $N\left(1+N_{g}^{2} N_{t}^{2}+N N_{g} N_{t}\right)$ flops, and the calculation of $\Psi=\mathbf{D}_{\mathbf{y}}^{H} \overline{\mathbf{P}}\left(N_{g}\right) \mathbf{D}_{\mathbf{y}}$ approximately requires $N^{2} N_{r}$ flops. For every single search loop of $\epsilon$, the computation of $\mathbf{a}^{H}(\epsilon) \Psi \mathbf{a}(\epsilon)$ demands $N\left(1+N+N N_{r}\right)$ flops. Consequently, the overall flops for the proposed scheme is approximated by:

$$
\begin{aligned}
\text { Flop }_{\text {Proposed }} & \approx \frac{1}{2} N N_{t}\left(1+N_{t}\right)\left[1+N_{g}\left(1+2 N_{g}\right)\right]+\frac{4}{3} N_{g}^{3} N_{t}^{3} \\
& +N\left[1+N_{g}^{2} N_{t}^{2}+N\left(N_{g} N_{t}+N_{r}\right)\right] \\
& +\mu N\left[1+N\left(1+N_{r}\right)\right],
\end{aligned}
$$

where $\mu$ denotes the number of search points. Similarly, the total flop count for the ML approach in (11) is approximated by:

$$
\begin{aligned}
\text { Flop }_{M L} & \approx \frac{1}{2} N N_{t}\left(1+N_{t}\right)[1+L(1+2 L)]+\frac{4}{3} L^{3} N_{t}^{3} \\
& +N\left[1+L^{2} N_{t}^{2}+N\left(L N_{t}+N_{r}\right)\right] \\
& +\mu N\left[1+N\left(1+N_{r}\right)\right] .
\end{aligned}
$$

As compared with (32), (31) shows that the proposed estimation seems to involve much more computational complexity than that of the ML scheme since $N_{g} \gg L$. Fortunately, because calculation of $\overline{\mathbf{P}}\left(N_{g}\right)$ is independent of the received data as well as the channel parameters, we can prestore the calculated $\overline{\mathbf{P}}\left(N_{g}\right)$ in the memory so

(a)

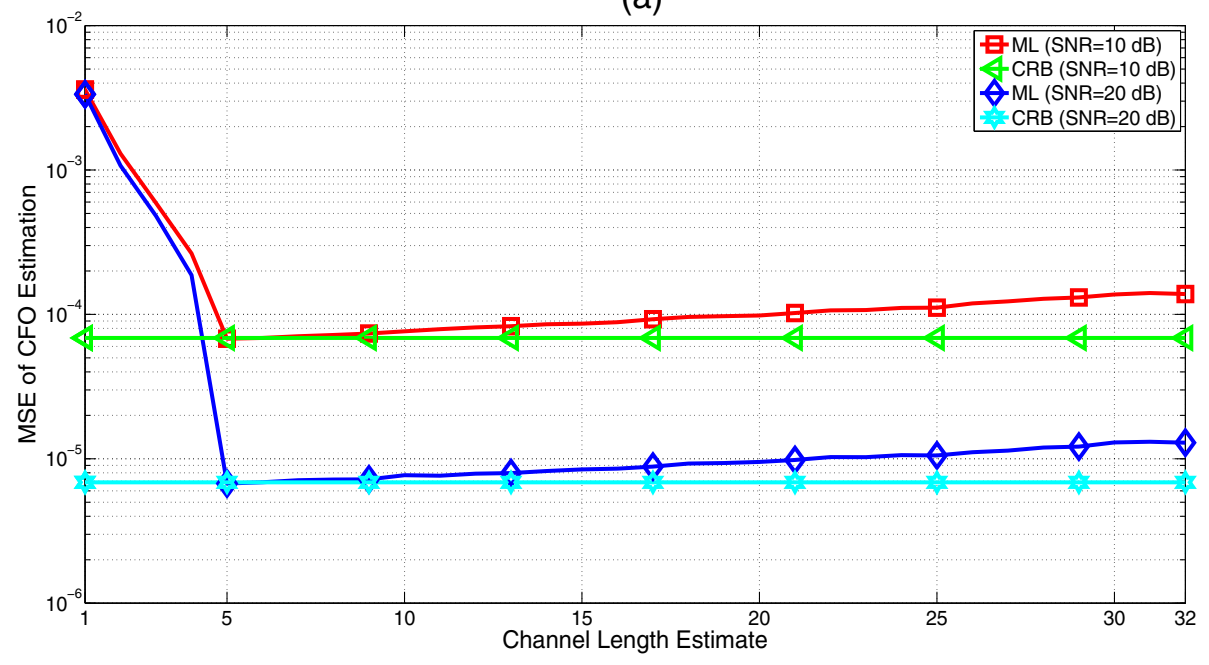

(b)

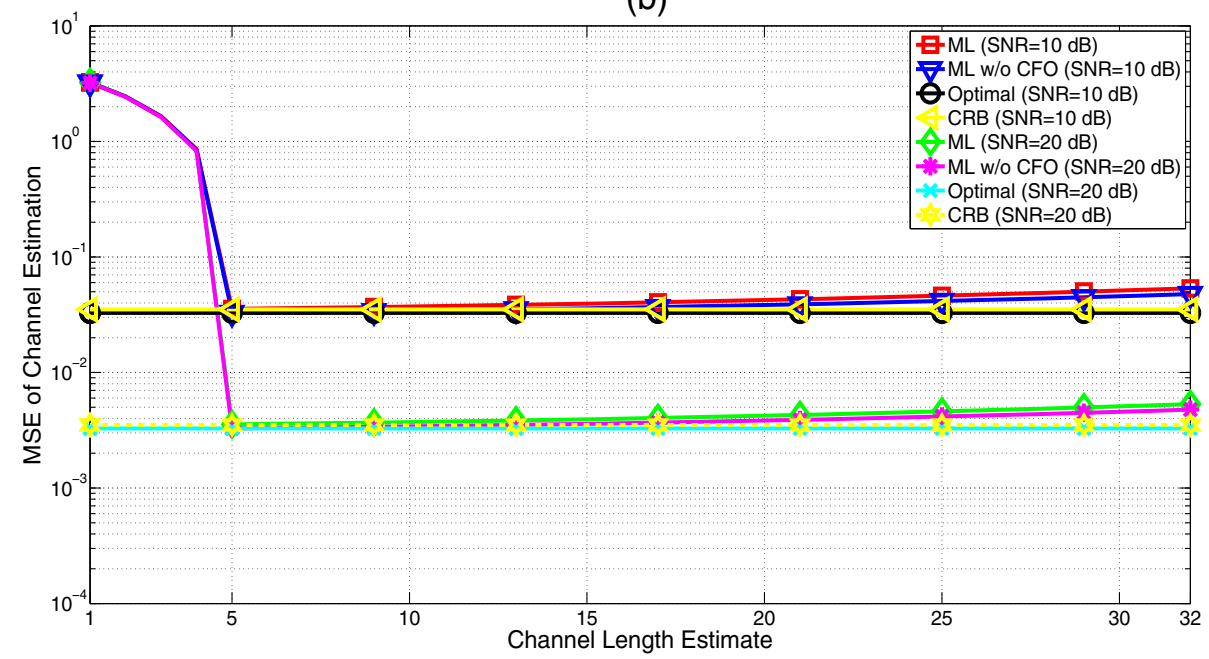

Figure 1 The MSE performance of the proposed estimation versus the channel length estimate $\hat{L}$ for the considered system. (a) MSE of CFO estimation; (b) MSE of the channel estimation. 
as to avoid the copious calculations. That is, we simply have to consider the count of flops of the computation of $\Psi$ and the search task in the coarse CFO estimation, and the computational complexity for the proposed scheme is thus reduced to:

$$
\text { Flop } \text { Proposed } \approx N^{2} N_{r}+\mu N\left[1+N\left(1+N_{r}\right)\right] .
$$

Here, we give a numerical example to demonstrate the comparison of complexity between two schemes. Given $N_{t}=N_{r}=2$ and $\mu=10^{4}$, Table 1 lists the computational complexity of the proposed and ML approaches in terms of flops for different sets of combination of $N, N_{g}$, and $L$, respectively. For $\left(N, N_{g}, L\right)=(64,16,1)$, the flop counts for the proposed and ML schemes are $1.240 \times 10^{8}$ and $1.235 \times 10^{8}$, respectively, and the percentage of the difference in this case is only $0.34 \%$. Furthermore, the maximum difference percentage in the table is under $1.5 \%$. It shows that the proposed scheme operates at the expense of an acceptably increased complexity as compared with the ML approach.

\section{Computer simulations}

Some simulation results have been conducted to verify the efficacy of the proposed CFO/channel estimator for a 128-subcarrier MIMO-OFDM system $(N=128)$, which is deployed with two transmit/receive antennas in a Rayleigh fading channel with channel length of $L=5$, where the channel impulse response is normalized such that $E\left\{\left\|\mathbf{h}_{i j}\right\|^{2}\right\}=1$. Here, the QPSK constellation is used in the data transmission where a CP of length $N / 4$ is inserted into the data stream. In the simulation mechanism, the CFO is uniformly and randomly generated from

(a)

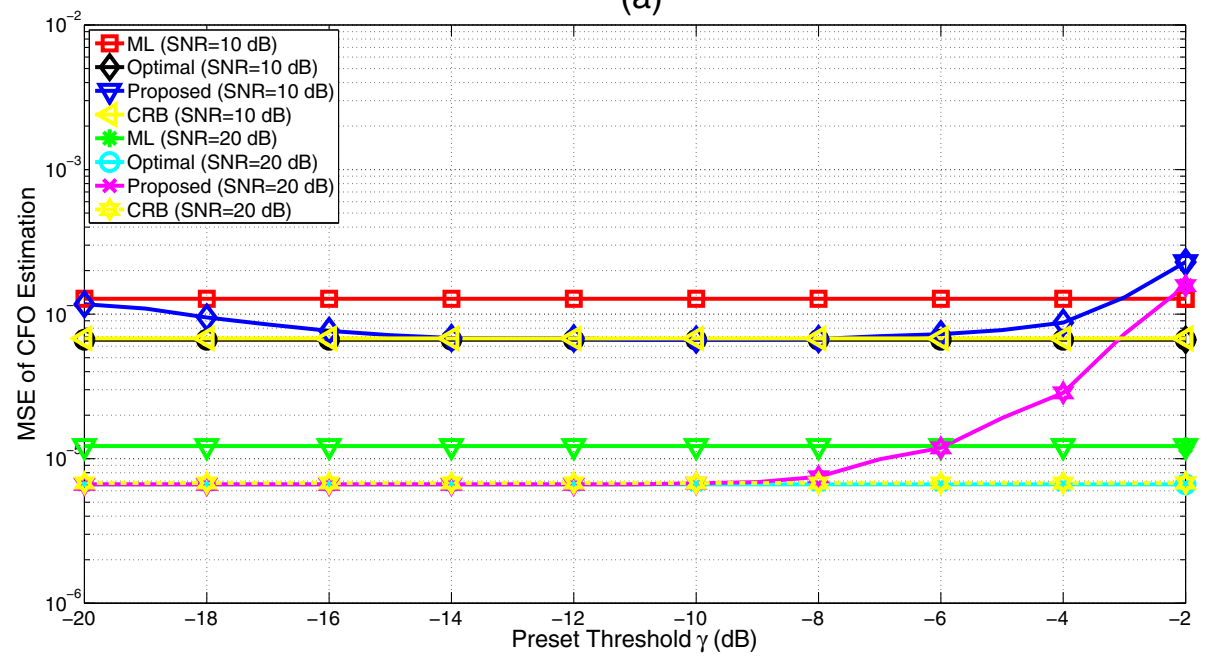

(b)

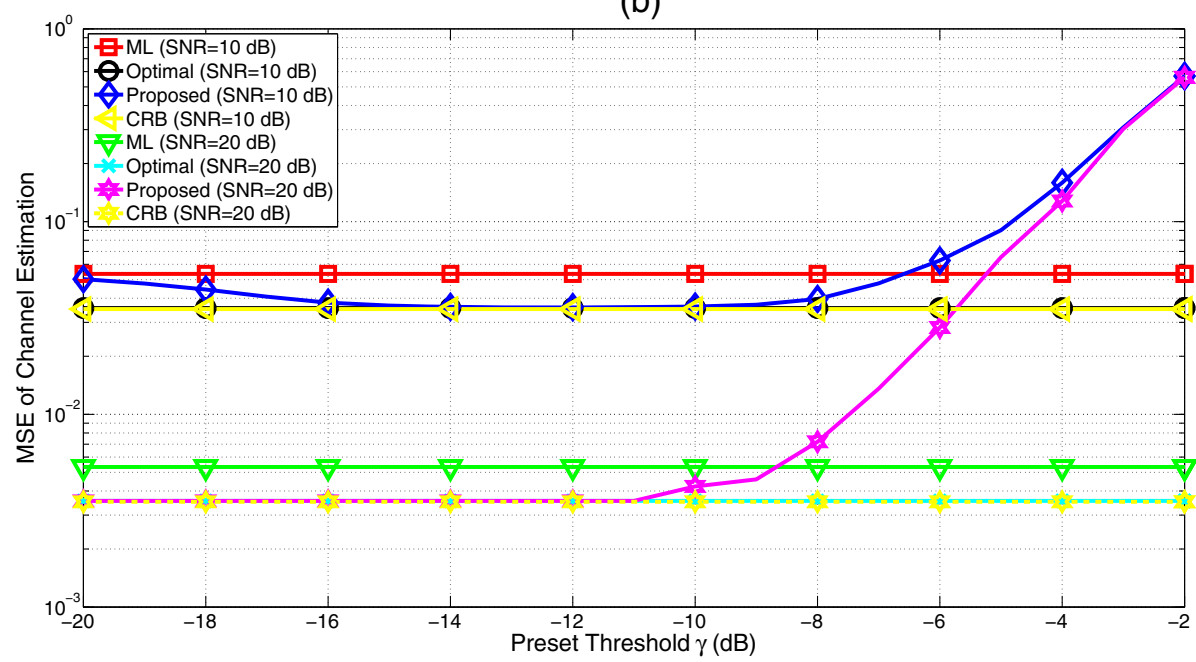

Figure 2 The MSE performance of the proposed estimation versus the preset threshold $\boldsymbol{\gamma}$ for the considered system. (a) MSE of CFO estimation; (b) MSE of the channel estimation. 
the interval $[-0.5,0.5]$. The SNR in decibel $(\mathrm{dB})$ is defined as SNR $=10 \log _{10}\left(E\left\{\|\mathcal{A}(L) \mathbf{h}\|^{2}\right\} / \sigma_{n}^{2}\right)$. To access the characteristic of the proposed estimator, the numerical results of the mean square errors (MSEs) of the CFO and channel estimation, which are defined by:

$$
\begin{aligned}
\operatorname{MSE}_{\epsilon} & =\frac{1}{Q} \sum_{q=1}^{Q}\left\|\hat{\epsilon}_{0}(q)-\epsilon_{0}\right\|^{2} ; \\
\operatorname{MSE}_{h} & =\frac{1}{Q} \sum_{q=1}^{Q} \sum_{j=1}^{N_{r}}\left\|\hat{\mathbf{h}}_{j}(q)-\mathbf{h}_{j}(q)\right\|^{2},
\end{aligned}
$$

are provided to evaluate the estimator's performance, where $Q$ is the number of independent trials. For comparison, means of the Cramer-Rao bounds (CRBs), which depend on the channel impulse response $\mathbf{h}$, are also included $[28,29]$ :

$$
\begin{aligned}
& \operatorname{CRB}(\epsilon)=\frac{\sigma_{n}^{2}}{2}\left[\mathbf{h}^{H} \mathcal{A}^{H}(L) \dot{\mathcal{E}}_{0}^{H} \overline{\mathbf{P}}(L) \dot{\mathcal{E}}_{0} \mathcal{A}(L) \mathbf{h}\right]^{-1}=\gamma \sigma_{n}^{2} \\
& \operatorname{CRB}(\mathbf{h})=\sigma_{n}^{2}\left\{\left[\mathcal{A}^{H}(L) \mathcal{A}(L)\right]^{-1}+\gamma \mathbf{z z}^{H}\right\},
\end{aligned}
$$

where:

$$
\begin{aligned}
\gamma & =\frac{1}{2}\left[\mathbf{h}^{H} \mathcal{A}^{H}(L) \dot{\mathcal{E}}_{0}^{H} \overline{\mathbf{P}}(L) \dot{\mathcal{E}}_{0} \mathcal{A}(L) \mathbf{h}\right]^{-1} \\
\mathbf{z} & =\left[\mathcal{A}^{H}(L) \mathcal{A}(L)\right]^{-1} \mathcal{A}^{H}(L) \dot{\mathcal{E}}_{0} \mathcal{A}(L) \mathbf{h} .
\end{aligned}
$$

Throughout the simulations, the granularity of the point search is given by $10^{-4}$ for the ML-based estimation. Each simulation point is obtained by taking the averages of all

(a)

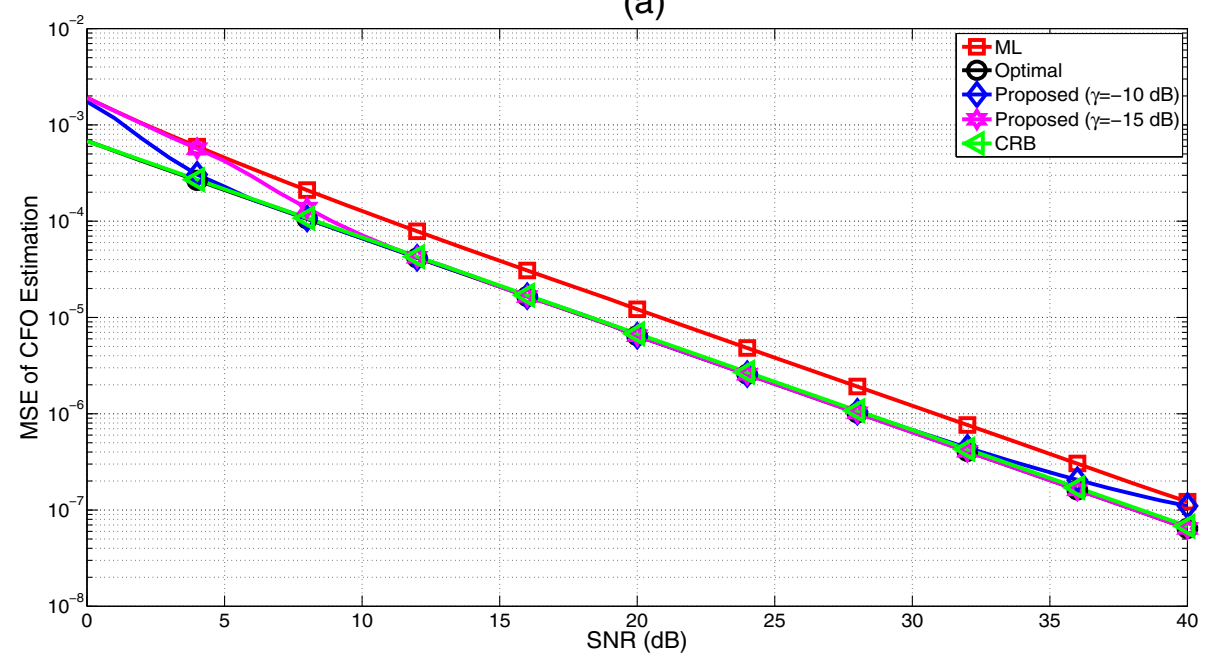

(b)

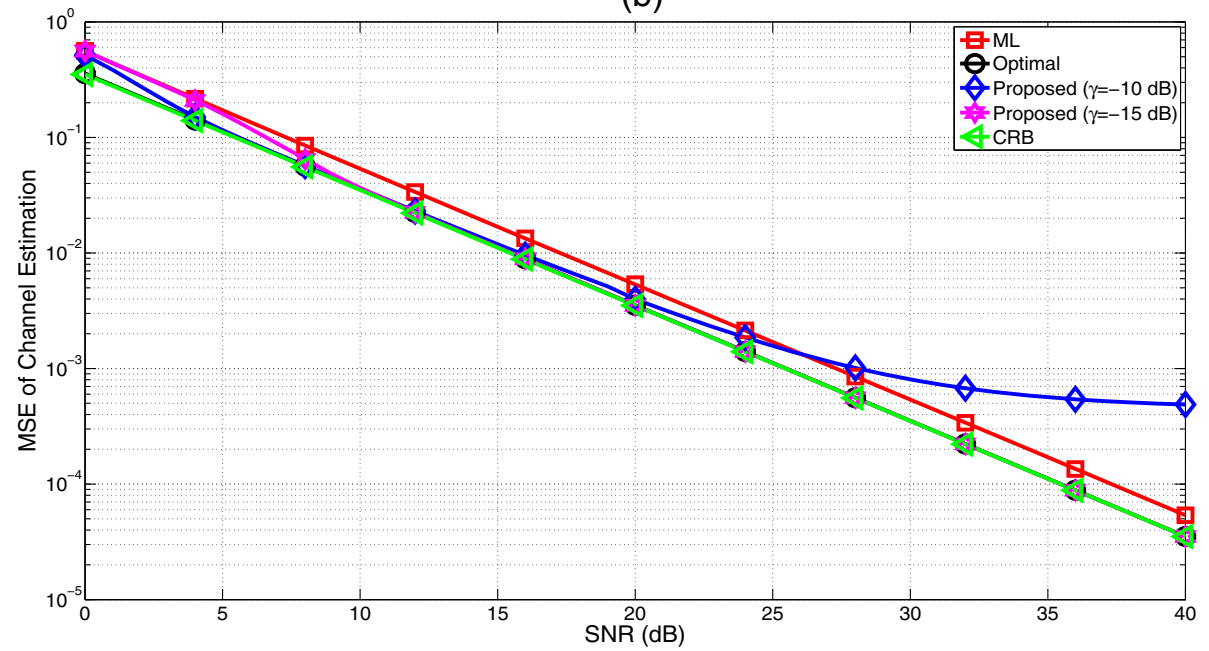

Figure 3 The MSE performance of the proposed estimation versus the input SNR for the considered system. (a) MSE of CFO estimation; (b) MSE of the channel estimation. 


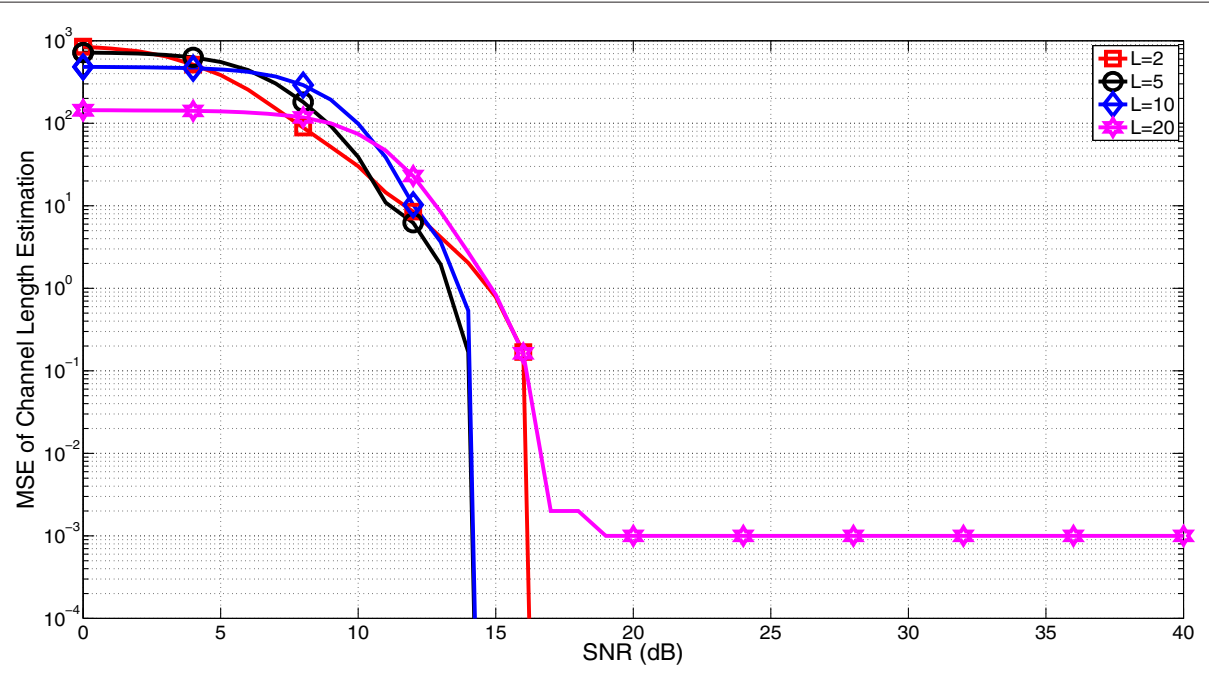

Figure 4 The MSE performance of the proposed CL estimation at different number of paths for the considered system.

independent trials, in which each trial uses a different set of the CFO, channel impulse response, and data/noise sequences.

In Figure 1, we investigate the effect of the estimation error in the channel length with different values of SNR. The MSE of the CFO versus CL estimate is plotted in Figure 1a. It shows that the MSE reaches a minimum value when channel length is correctly used $(\hat{L}=5)$. In regard to the under-determined case, the ML estimator in (11) is not able to provide a reliable performance. This is because the degree of freedom is not sufficiently large for reconstructing the received signal, which results in an erroneous cost function expressed by (7). A reliable CFO estimate cannot be obtained by searching the minimum of the erroneous cost function. For $\hat{L}>L$, the MSE in
CFO increases by the value of $\Delta L=\hat{L}-L$. Furthermore, we examine the MSE performance of the channel estimation while varying CL estimate. For comparison, the simulation results associated with the ML estimator without CFO are also included. The corresponding results shown in Figure 1b and Figure 1a have a similar tendency. As expected, the MSE performance is slightly degraded owing to CFO.

In Figure 2, we examine the impact of the preset threshold $\gamma$ on the estimate performance for SNR $=10$ and $20 \mathrm{~dB}$. For comparison, the results obtained with the ML estimator using the $\mathrm{CP}$ length and true $\mathrm{CL}$ are also included and represented by ML and optimal, respectively. This figure indicates that the proposed estimation approaches the optimal case when $\gamma$ is sufficiently small

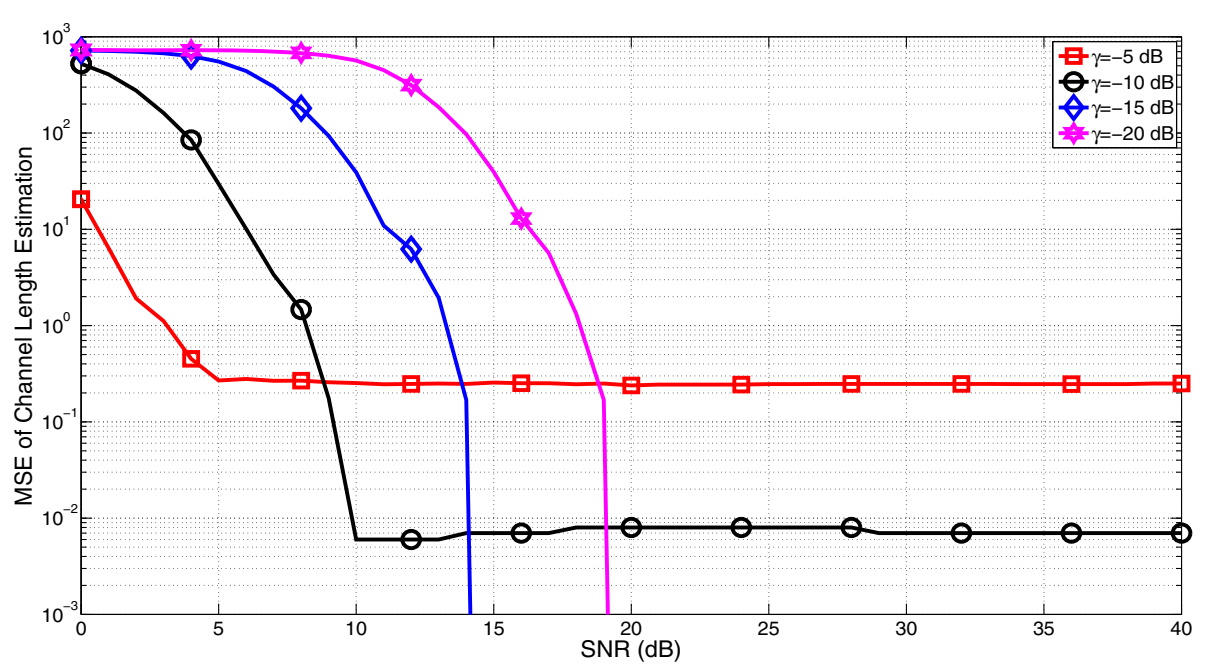

Figure 5 The MSE performance of the proposed CL estimation at different preset threshold, $\gamma$, for the considered system. 
$(-15 \mathrm{~dB}<\gamma<-10 \mathrm{~dB})$. For a large preset threshold, the proposed scheme substantially degrades since the meaningful paths are not successfully detected. There is a large miss probability in the detection of the channel response, which prevents a reliable CFO and channel estimation. It is noteworthy that deep faded paths are filtered by a large preset threshold $\gamma$, especially for high SNR. On the contrary, for $\mathrm{SNR}=10 \mathrm{~dB}$, a performance degradation occurs as the preset threshold is very small $(\gamma=-20 \mathrm{~dB})$ owing to a large false alarm in detecting the channel response. It concludes that a proper choice of $\gamma$ is essential in the presence of the CFO. Since the impact of the performance caused by a path signal which is much less than $-10 \mathrm{~dB}$ is slight when compared with that caused by the dominant path, the acceptable range of SNR in this case is about $-20<\gamma<-10 \mathrm{~dB}$.
In Figure 3, we investigate the impact of the SNR on the proposed CFO/channel estimator with $\gamma=-10$ and $-15 \mathrm{~dB}$. From Figure $3 \mathrm{a}$, it can be shown that the MSE decreases as the value of SNR increases. In addition, using the preset threshold $\gamma=-15 \mathrm{~dB}$, the proposed scheme provides a performance similar to that of the ML estimator with a prior knowledge of channel length. The result verifies the efficiency of the proposed CFO estimation. For low SNR, a large false alarm introduced by noise drops the MSE performance in the CFO estimation. Figure $3 \mathrm{~b}$ shows the MSE performance of the channel estimation. It is observed that the estimator's performance can be improved by increasing the SNR. The proposed scheme with CL estimation achieves a performance similar to that of the optimal estimation for $\gamma=-15 \mathrm{~dB}$. However, in the case of $\gamma=-10 \mathrm{~dB}$, the proposed estimator fails to offer a

(a)

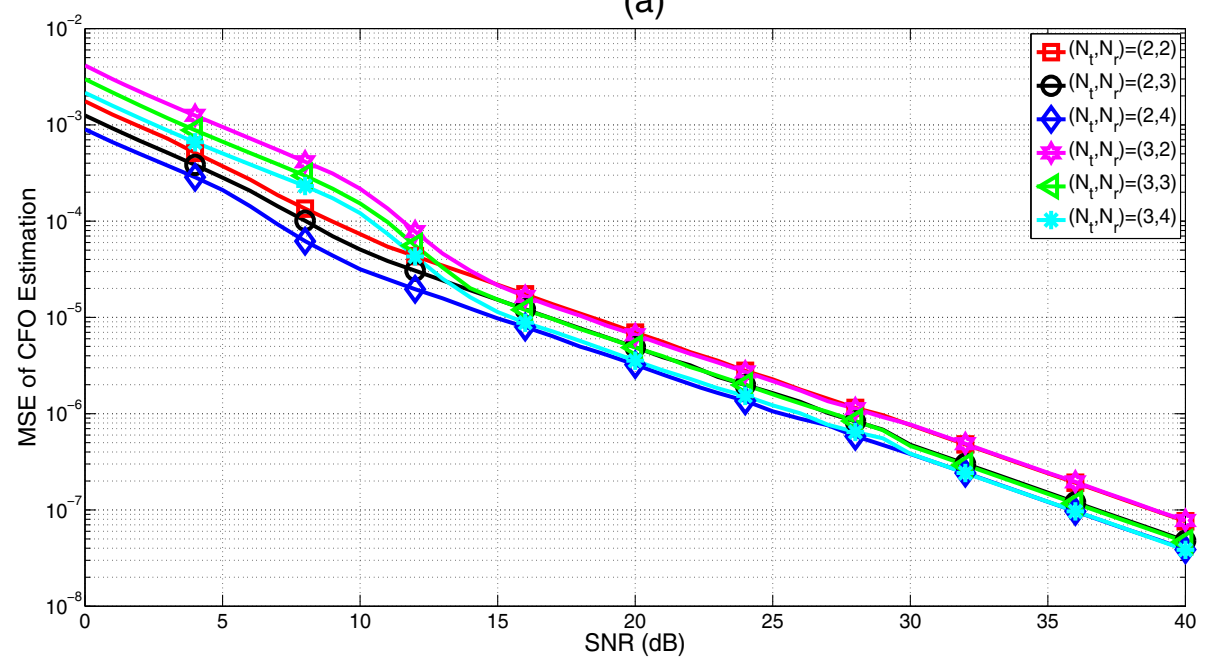

(b)

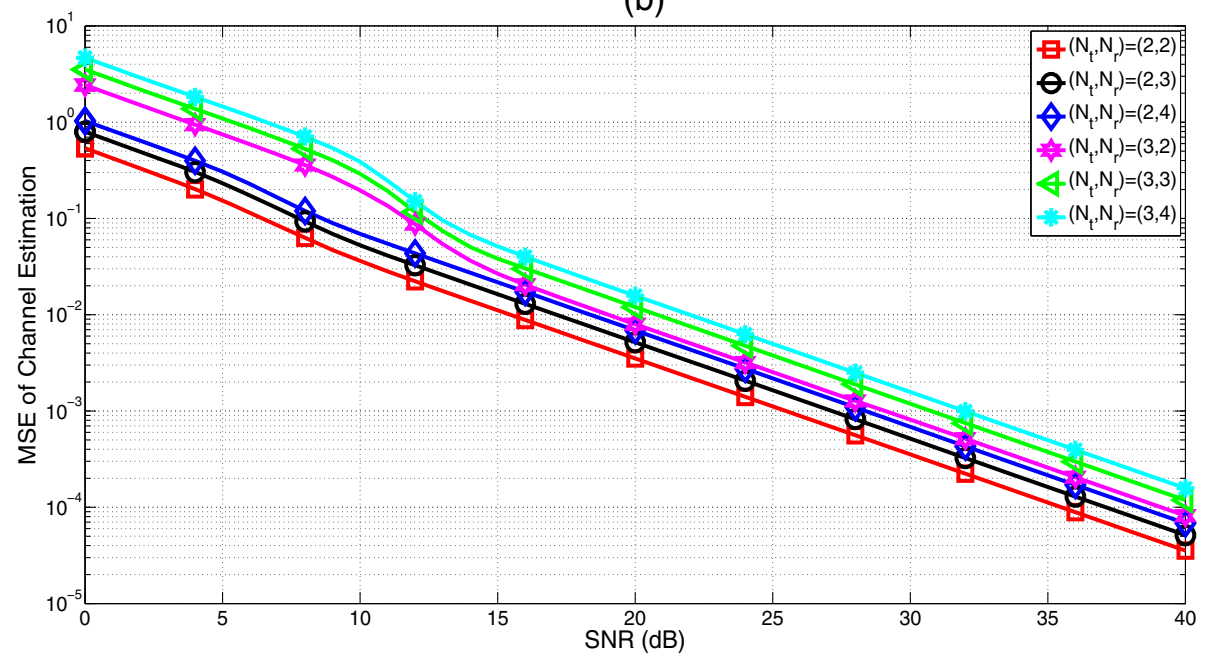

Figure 6 The MSE performance versus the input SNR for the considered system with different numbers of transmit/receive antennas. (a) MSE of CFO estimation; (b) MSE of the channel estimation. 
performance comparable to that of the optimal case while the SNR is getting higher and higher. This is because the proposed CL estimator exhibits under-determining due to a deep fading.

In Figure 4, we present the MSE of CL estimation versus SNR for a set of different $C L$ values while setting the preset threshold $\gamma=-15 \mathrm{~dB}$. It can be shown that in the figure the MSE of the proposed CL estimation suddenly approaches to zero while the SNR is greater than 15 $\mathrm{dB}$. On the contrary, when the SNR is smaller than $10 \mathrm{~dB}$, large noise power results in over-determination and thus significantly degrades the MSE performance of CL estimation. In a rich multipath environment $(L=20)$, the weak paths introduced by deep fading are removed because they can be treated as noise while the path number is being determined. This results in the under-determined problem.

In Figure 5, we illustrate the impact of the preset threshold $\gamma$ on CL estimation. Figure 5 shows the MSE versus SNR for different values of $\gamma$. Given $\gamma=-15$ or $-20 \mathrm{~dB}$, the proposed $\mathrm{CL}$ estimator reaches $\mathrm{CL}$ perfectly when SNR is greater than $15 \mathrm{~dB}$. On the contrary, when $\gamma>-10 \mathrm{~dB}$, the miss probability increases and the underdetermination is brought about owing to the weak paths caused by deep fading being punctured. For the underdetermination cases, there are error floors even when the SNR is increased.

Figure 6a plots the MSE curves of CFO versus SNR for several sets of $\left(N_{t}, N_{r}\right)$. It shows that the MSE performance of CFO can be improved by increasing the receive diversity as $N_{t}$ is given. When SNR is low, a little degradation in the MSE performance is induced because fewer degrees of freedom are provided for the minimization problem in (5). Figure 6b shows the MSE performance of the channel estimation. Given $N_{t}$, the MSE performance degrades as $N_{r}$ increases because the larger the size of $\mathbf{h}$ is, the poorer the performance of channel estimation will be.

\section{Conclusions}

An algorithm for the joint $\mathrm{CFO}$ and channel estimation is presented for the MIMO-OFDM scenario. As compared with the conventional ML estimate approach, the novelty of the proposed algorithm is that it does not require to know the channel length in advance. Furthermore, we derived a set of beneficial analyses for the MSE of channel length estimate, which can be further applied to the conventional ML estimator. Analytic and simulation results for the proposed estimate technique have been presented where the prior knowledge of channel length is not used because channel length estimate can be reached in our scheme simultaneously. It can be shown that the proposed scheme offers a performance comparable to that of the optimal ML-based estimator which operates well only when perfect channel length is assumed. The feasibility of implementation for the proposed approach is ensured by the presented complexity analyses.

\section{Competing interests}

The authors declare that they have no competing interests.

\section{Acknowledgements}

This works was supported in part by the Ministry of Science and Technology under Grand MOST 103-2221-E-239-013.

\section{Author details}

1 Department of Electronic Engineering, National United University, Miaoli 36003, Taiwan. 2 Department of Optoelectronics and Communication Engineering, National Kaohsiung Normal University, Kaohsiung 82444, Taiwan.

Received: 2 October 2014 Accepted: 3 March 2015

Published online: 20 March 2015

\section{References}

1. O Besson, P Stoica, On parameter estimation of MIMO flat-fading channels with frequency offsets. IEEE Trans. Signal Process. 51(3), 602-613 (2003)

2. H Sampath, S Talwar, J Tellado, V Erceg, A Paulraj, A fourth generation MIMO-OFDM broadband wireless system design, performance, and field trial results. IEEE Commun. Mag. 40(9), 143-149 (2002)

3. BHu X Wang, A low-complexity ML estimator for carrier and sampling frequency offsets in OFDM systems. IEEE Commun. Lett. 18(3), 503-506 (2014)

4. NL Hung, LN Tho, CC Ko, Turbo processing for joint channel estimation, synchronization, and decoding in coded MIMO-OFDM systems. EURASIP Wireless Commun. Netw (2009). doi:10.1155/2009/206524

5. H Minn, M Al-Dhahi, Y Li, Optimal training signals for MIMO OFDM channel estimation in the presence of frequency offset and phase noise systems. IEEE Trans. Commun. 54(10), 1754-1759 (2006)

6. Y Jin, XG Xia, An interference nulling based channel independent precoding for MIMO-OFDM systems with insufficient cyclic prefix. IEEE Trans. Commun. 61(1), 131-143 (2012)

7. X Ge, X Huang, Y Wang, M Chen, Q Li, THan, C-X Wang, Energy-efficiency optimization for MIMO-OFDM mobile multimedia communication systems with QoS constraints. IEEE Trans. Veh. Technol. 63(5), 2127-2138 (2014)

8. OH Salim, AA Nasir, H Mehrpouyan, W Xiang, S Durrani, RA Kennedy, Channel, phase noise, and frequency offset in OFDM systems: joint estimation, data detection, and hybrid Cramer-Rao lower bound. IEEE Trans. Commun. 62(9), 3311-3325 (2014)

9. H Wang, XG Xia, Q Yin, Distributed space-frequency codes for cooperative communication systems with multiple carrier frequency offsets. IEEE Trans. Wireless Commun. 8(2), 1045-1055 (2009)

10. S Rahimi, B Champagne, Joint channel and frequency offset estimation for oversampled perfect reconstruction filter bank transceivers. IEEE Trans. Commun 62(6), 2009-2021 (2014)

11. YCWu J Chen, S Ma, TS Ng, Joint CFO and channel estimation for multiuser MIMO-OFDM systems with optimal training sequences. IEEE Trans. Signal Process. 56(8), 4008-4019 (2008)

12. M Ghogho, A Swami, Training design for multipath channel and frequency-offset estimation in MIMO systems. IEEE Trans. Signal Process. 54(10), 3957-3965 (2006)

13. Z Zhang, W Zhang, C Tellambura, MIMO-OFDM channel estimation in the presence of frequency offset. IEEE Trans. Wireless Commun. 7(6), 2329-2339 (2008)

14. M Dong, L Tong, Optimal design and placement of pilots symbols for channel estimation. IEEE Trans. Signal Process. 50(12), 3055-3069 (2002)

15. Y Zeng, AA Leyman, TS Ng, Joint semiblind frequency offset and channel estimation for multiuser MIMO-OFDM uplink. IEEE Trans. Commun. 58(9), 2697-2708 (2010)

16. KJ Kim, MO Pun, RA Iltis, Joint carrier frequency offset and channel estimation for uplink MIMO-OFDMA systems using parallel Schmidt Rao-Blackwellized particle filters. IEEE Trans. Commun. 55(12), 2270-2278 (2007)

17. R Jose, KVS Hari, Maximum likelihood algorithms for joint estimation of synchronisation impairments and channel in multiple input multiple 
output-orthogonal frequency division multiplexing system. IET Commun. 7(15), 1567-1579 (2013)

18. E Manasseh, S Ohno, M Nakamoto, in Proc. of Asia-Pacific Signal and Information Processing Association Annual Summit and Conference (APSIPA ASC 2011), Oct 18-21. Preamble based joint channel and CFO estimation for MIMO-OFDM systems with null subcarriers (Xi'an, China, 2011)

19. H Akaike, A new look at the statistical model identification. IEEE Trans. Autom. Control. AC19(6), 716-723 (1973)

20. M Krondorf, TJ Liang, R Irmer, G Fettweis, in Proc. Wireless Conf. April 2-5. Improved channel estimation for complexity-reduced MIMO-OFDM receiver by estimation of channel impulse response length (Athens Greece, 2006), pp. 1-6

21. DKC So, RS Cheng, Iterative EM receiver for space-time coded systems in MIMO frequency-selective fading channels with channel gain and order estimation. IEEE Trans. Wireless Commun. 3(6), 1928-1935 (2004)

22. J Siew, J Coon, A Nix, M Beach, S Armour, J McGeehan, in Proc. Commun. Wireless Syst. Conf. Sept 20-22. A joint channel and channel length estimation algorithm for MIMO-OFDM (Mauritius, 2004), pp. 285-289

23. A Tomasoni, G Gatti, S Bellini, M Ferrari, M Siti, Efficient OFDM channel estimation via an information criterion. IEEE Trans. Wireless Commun. 12(3), 1352-1362 (2013)

24. J Via, I Santamaria, J Perez, Effective channel order estimation based on combined identification/equalization. IEEE Trans. Signal Process. 54(9), 3518-3526 (2006)

25. M Greenberg, Advanced Engineering Mathematics, 2nd edn. (Prentice Hall, London, UK, 1997)

26. TC Chen, $T T$ Lin, Blind carrier frequency offset mitigation in space-time block-coded multicarrier code division multiple access uplink transmission. Intl. J. Commun. Syst (2014). doi:10.1002/dac.2823

27. GH Golub, CF Van Loan, Matrix Computation, 3rd edn. (Johns Hopkins University Press, Baltimore, MD, 1996)

28. P Stoica, O Besson, Training sequence design for frequency offset and frequency-selective channel estimation. IEEE Trans. Commun. 51(11), 1910-1917 (2003)

29. S Salari, Ardebilipour, M Ahmadian, Jion for multiple-input multiple-output-orthogonal frequency-division multiplexing systems. IET Commun. 2(8), 1069-1076 (2008)

\section{Submit your manuscript to a SpringerOpen journal and benefit from:}

- Convenient online submission

- Rigorous peer review

- Immediate publication on acceptance

- Open access: articles freely available online

- High visibility within the field

- Retaining the copyright to your article

Submit your next manuscript at $\boldsymbol{\triangleright}$ springeropen.com 